\title{
Resistance Training Reduces Pain Indices and Improves Quality of Life and Body Strength in Women with Migraine Disorders
}

\section{Payam Sari Aslani}

Kermanshah University of Medical Sciences

Maryam Hassanpour

Razi University of Kemanshah: Razi University

Omid Razi

Razi University of Kemanshah: Razi University

\section{Beat Knechtle}

University of Zurich: Universitat Zurich

Abdolhossein Parnow ( $\nabla$ parnowabdolhossein@gmail.com )

Razi University of Kemanshah: Razi University https://orcid.org/0000-0003-1965-2626

\section{Research Article}

Keywords: Women, Resistance Training, Migraine, Headache Indices, Quality of Life

Posted Date: October 1st, 2021

DOl: https://doi.org/10.21203/rs.3.rs-573627/v1

License: (9) This work is licensed under a Creative Commons Attribution 4.0 International License. Read Full License 


\section{Abstract \\ Purpose}

The benefits of resistance training (RT) for migraineurs appears to be lacking although beneficial of aerobic training have been shown in previous studies. The aim of current study, therefore, is to investigate the influence of RT on migraine headache indices, upper and lower-body strength, and quality of life (QOL) in women with migraine disorders.

\section{Methods}

Twenty female migraine patients who were referred by a neurologist were randomly assigned in two groups including RT group $(n=10)$ and control $(C O N)$ group $(n=10)$. The RT protocol consisted of 8 weeks, 3 sessions per week, 45-60 min per session. Visual Analog Scale (VAS) and pain diary were utilized to measure the subjects' migraine indices including duration, pain severity, and frequency within $48 \mathrm{~h}$ pre- and post-training protocol. Patients in Control group (CON) has been refrained from regular exercise during this study. The quality of life (QOL) and muscular strength were measured by the Headache Impact Tests (HIT) and one-repetition maximum (1RM) test, respectively, for $48 \mathrm{~h}$ pre- and posttraining protocol.

\section{Results}

The RT resulted in a significant decrease in the migraine indices (headache intensity, frequency, and duration) ( $p=0.02, p=0.001, p=0.04$, respectivetly). Increased quality of life and chest and leg muscular strength significantly ( $p=0.001$ for all) were also showed after 8-week RT protocol. However, there was not any significant differences between groups considering, BMI and waist-hip ratio (WHR) after executing RT protocol $(p>0.05)$.

\section{Conclusion}

Based on results of current study that mainly had a positive trend, it could state that RT protocol with special considering for migraine patients is probably an alternative therapy or augmentative complement to established interventions for migraine treatment.

\section{Introduction}

Most people with chronic illnesses or disabilities are less physically active than their healthy counterparts. The individuals' physical activity could be severely reduced by chronic diseases or disabilities, resulting in physical weakness due to physiological disorders $(1,2)$. Research shows that physical activity not only protects the health but also prevents the disease $(2,3)$. The migraine patients 
are usually recommended to perform the exercise training $(2,3)$ since regular exercise contributes to the reduction of headache attacks frequency $(4,5)$. The results indicated that the patients did not have sufficient physical activity in form of well-designed exercise training in comparison with a healthy control group (3).

Migraine is a debilitating neurovascular disorder characterized by severe headache attacks often associated with autonomic and neurological symptoms and it is normally instantiated as an attack enduring from four to $72 \mathrm{hrs}$. The distinctive features of this attack include unilateral headaches with pulsating quality, moderate or severe intensity, and aggravation by routine physical activity (not welldesigned exercise training), and associated nausea, photophobia, and phonophobia $(6,7)$. The debilitating features of migraine usually lead to chronic fatigue, psychosocial disorders, depression, and significant loss of productivity, social involvement, and quality of life $(\mathrm{QOL})(8,9)$. Because many of the routinely prescribed medications have inadequate efficacy and significant adverse effects, alternative treatment regimens are under investigation $(8,9)$.

It is well-accepted that physical activity is an influential reaction to the medication of many disorders. Accordingly, physical activity is largely an effective strategy for migraine treatment(8). Moreover, the relevant literature documents various studies reporting the positive effects of aerobic exercise on migraine indices (e.g., pain intensity, frequency, and duration), cardiovascular endurance, and QOL $(4,7$, 10-13). The majority of these investigations report variation in calcitonin gene-related peptide (CGRP) levels and Endogenous Opioids, particularly in beta-endorphins, as the reason for this improvement $(4,8$, 14), althugh Roy and et al in systematic aand metanalysis review reported low to moderate evidence of aerobic training to decrease pain intensity, frequency and duration of migraine in people with migraine disorders (15).

Although in a review research recently has been reported effects of different training (aerobic and anaerobic training) are insufficient to make any recommendations (16), no research has focused on resistance training (RT) in the literature of migraine and exercise. It is a high significance to elaborate on the effect of RT which is considered as a safe and prevalent sort of training on migraine; hence, the primary purpose of the current investigation was to understand the impact of RT on patients suffering from migraine headache. As mentioned previously, to the best knowledge of the researchers, there is currently no evidence of RT and possible changes or contributions it may bring to migraine indices as well as QOL. Given this issue in mind, thus, as research further indicates that more than $10 \%$ of the world population suffers from migraine headaches, of which $15-18 \%$ are females (6-9\% are males) (6), this study designed on women and investigated the effect of RT on migraine indices, upper and lower body muscle strength, and QOL in females with migraine disorders. The research hypothesis was that an eightweek RT program can influence/impact/effect on migraine headache indices, body strength, and QOL in female patients' agonizing pain of migraine disorders. In other words, resistance training program has no influence/impact/effect on pain indices, body strength, and quality of life in women with migraine disorders. 


\section{Method}

\section{Research design}

This was a randomized controlled clinical trial (RCT) with a semi-experimental design (two groups in the form of pre-test and post-test) (RCT code: IRCT2015100910824N2) and was conducted among the migraine patient's community from 2015 to 2018 in Kermanshah city, Iran. The experimental procedures and study protocols were approved by the Ethics Committee of Kermanshah University of Medical Sciences (ethical code: kums.rec.1394.15, 2015), Kermanshah, Iran.

In order to determine the number of subjects, the randomized sampling method was used. More than 50 untrained female migraines patients voluntarily were recruited from neurology clinics, and neurology center of Kermanshah hospital (Kermanshah, Iran). The criteria proposed and outlined by the International Headache Society (IHS) (International Classification of Headache Disorders (ICHD)) were utilized to provide the pertinent diagnosis. Inclusion criteria was including: (a) age range from 18 to 45 years; (b) migraine with or without aura with a frequency of 2-8 attacks per month confirming by the neurologist; (c) Not having a history of regular exercise in the last three months, (d) having no chronic desiases such as cardiovascular disease, hypertension, diabetes, arthritis, cancer, asthma, AIDS, meningitis and Multiple Sclerosis. Exclusion criteria was including: (a) situations that researchers have understood during research period such as pregnancy, breastfeeding, abuse of drugs or alcohol; (b) involving in any extra exercise training programs; (c) not interested to continue or change in personal life schedule. Finally, according to the study criteria for subject selection, 20 untrained female migraine patients were selected by the neurologist and randomly assigned in two groups: control (CON) $(n=10)$ and resistance training (RT) $(n=10)$. In order to estimate the number of participants needed in the study, a sample size calculation was performed using G*Power Software version 3.1.9.6 (Düsseldorf, Germany) for a repeated measures ANOVA (within factors), using an a rejection criterion of 0.05 and 0.95 (1- $\beta$ ) power, and an average correlation of rho $=0.50$ between the repeated measures for 2 within subject measurements to detect a large effect $(f=0.6)$. The power calculation indicated that a minimum of 10 participants was required to be able to find such an effect (17). All patients were informed about the project (collection of data, take measurements, exercise protocol and duration of the study) and requested to fill out the consent form.

Patients in CON group were instructed to keep a diary regarding exercise during the experiments period. Experimental groups, included RT, executed resitance training (see below). Both two groups received their drug treatment under neurologist supervision. In addition, $24 \mathrm{~h}$ before and $48 \mathrm{~h}$ after the training protocol, body composition factors (height, age, weight, fat percentage) (In body; Model: Zuse 9.9, South Korea), body mass index (BMI), waist-to-hip ratio (WHR), QOL, mograine status chest and leg strength were measured. The general demographic characteristics of the participants at the baseline were shown in Table 1. 
Table 1

Demographic data of the patients (Mean $\pm S D$ )

\begin{tabular}{|llllll|}
\hline $\begin{array}{l}\text { Variables } \\
\text { groups }\end{array}$ & Age (year) & Height $(\mathrm{cm})$ & Weight $(\mathrm{kg})$ & WHR $(\mathrm{cm})$ & $\mathrm{BMI}\left(\mathrm{kg} / \mathrm{m}^{2}\right.$ \\
\hline CON & $30.10 \pm 7$ & $159.80 \pm 3.82$ & $60.07 \pm 9.30$ & $0.76 \pm 0.03$ & $23.04 \pm 4.15$ \\
\hline EXP & $30.25 \pm 6.10$ & $161.75 \pm 6.32$ & $64.11 \pm 13.18$ & $0.75 \pm 0.04$ & $25.03 \pm 4.43$ \\
\hline
\end{tabular}

\section{Resistance Training (RT) Program}

The RT program was comprised of a period of 8 weeks that was executed 3 time per week, $45-60$ min in session including a $15 \mathrm{~min}$ warm up (i.e. $5 \mathrm{~min}$ of jogging/ 5 min stretching / 5 min of weightlifting, including a set of 10 to 12 repetitions at intensity $40 \%$ of $1 \mathrm{RM}$ ) followed by a $30-45$ min main RT program period (i.e., five movements: arm pull down, arm pullover, sit up, leg extension and leg curl) (presented in Table 1) and a 5- min cool-down period (i.e., active cooling and stretching movements). During the training session, music was included to make the program more pleasant. The frequency and intensity of training (determined \% of 1RM) were based on recommendations for increasing body strength from the National Strength Conditioning Association (NSCA) and the authors' experience of RT. In addition, it should be noted that 2 weeks of familiarity was considered before starting RT program.

Table 2

Resistance training protocol

\begin{tabular}{|lllllllll|}
\hline Weeks of training & $\mathbf{1}$ & $\mathbf{2}$ & $\mathbf{3}$ & $\mathbf{4}$ & $\mathbf{5}$ & $\mathbf{6}$ & $\mathbf{7}$ & $\mathbf{8}$ \\
\hline Set & 2 & 3 & 3 & 3 & 3 & 3 & 3 & 3 \\
\hline Repetition & 15 & 12 & 12 & 10 & 10 & 10 & 8 & 8 \\
\hline Rest (second) & 60 & 60 & 60 & 60 & 60 & 60 & 60 & 60 \\
\hline Intensity(\%1RM) & 45 & 50 & 50 & 60 & 60 & 70 & 70 & 75 \\
\hline
\end{tabular}

\section{Assessments}

One repetitions maximum (1RM), migraine indices (i.e., pain intensity, frequency, and duration), quality of life, body mass index (BMI), and waist to hip ratio (WHR) were evaluated at 48 hours pre- and posttraining program. Prior to beginning RT program, 48 hours, 1RM was determined for all five movements mentioned above as will explain in next section. 1RM determination was repeated after four weeks for monitoring training load as body strength could increase in the short time.

\section{One repetition maximum (1RM)}

$1 \mathrm{RM}$ in RT, in form of weight training, is the maximum amount of weight that a person can possibly lift for one repetition (18). it realy is a method to assessment maximual force that produced by a muscle or a group of muscles. 1RM should be calculated to determine workload percentage and monitoring progression during RT program. 1RM calculates generally in direct (maximal) and indirect (submaximal) 
methods (18). In direct way, participant will finally reach to maximum force produced by muscle groups. This way is not safe for unprofessional people. The indirect (submaximal) was used to determine 1RM in migraine patients who had less evening for inexperienced exercisers as well as indirect method is preferred as it is safer, quicker (18). 1RM test was monitored by experienced coach. Finally, By using Brzycki formula (below) (19), 1RM was calculated.

$1 \mathrm{RM}=($ weight lifted) $/[1.0278-$ (repetitions $\times 0.0278)](19)$. Where the repetitions would be less than 10 repetitions.

\section{Migraine Status}

The patients were instructed on how to keep migraine diaries regarding migraine attacks (headache frequencies), days with migraine (headache duration), and the daily average migraine pain (headache intensity) during four weeks pre- and post-training program. The diary was based on the VAS which consisted of a 100-mm straight line with "no pain" as one endpoint and "worst imaginable pain" as the other (4).

\section{Quality of Life (QOL)}

QOL was assessed using the headache impact test (HIT-6) questionnaire. It consists of six questions. HIT is regarded as a measurement device to obtain the possible influence the migraine headaches may impose on one's physical performance on the job, at school, at home, and in social contexts. The obtained score could illustrate the extent of the impact that migraine headaches direct on patients' normal daily life and their proficiency to function. HIT was developed by an international team of headache experts from neurology and primary care medicine in collaboration with the psychometricians who developed the SF-36® health assessment tool. This questionnaire was designed to help the patients describe and communicate the way they feel and what they cannot do due to headaches (20).

\section{Body mass index (BMI)}

The BMI is defined as the body mass divided by the square of the body height and is universally expressed in units of $\mathrm{kg} / \mathrm{m}^{2}$, resulting from mass in kilograms and height in meters (21). In this study, metal tape and weighing scale (support scale SSB- 215 D model, made in Korea) were used to measure body height and body weight. In order to measure body height, the subjects were requested to take off their shoes, undress their bulky clothing, and remove their ornaments; they were also asked to stand with feet flat together, legs straight, arms at sides, shoulders at level, and against the wall. To set out the right angle with the wall, researchers used a flat headpiece; then, they specified the spot on which the bottom of the headpiece meets the wall. After that, a metal tape was used to measure the height from the base, on the floor, to the marked measurement on the wall. Next, their weight was recorded. Finally, the obtained height and weight data were put into the following equation to assess BMI for each subject: Weight $(\mathrm{kg})$ / Height $(m)^{2}$.

\section{Waist-hip ratio (WHR)}


Waist-hip ratio (WHR) is the dimensionless ratio of the circumference of the waist to that of the hips. WHR is calculated as waist measurement divided by the hip measurement. Meter tape was used to measure the hip circumference at its widest point and waist circumference at the narrowest point. To these, the subjects were requested to stand with feet close together, arms at the side, and body weight, evenly distributed with minimal clothing. The measurements were performed at the end of normal respiration. Each measurement was repeated twice.

\section{Drug administration}

Based on supervision of expert neurologist, participants used and monitored migraine drug During present study. According on initial assessment, needed dose of drug (including Nortriptyline and Duloxetine, Diclofenac Sumatriptan, Propranolol, Depakin, Dexamethazone, Gabapentin, Ergotamine, Venlafaxine, Zolpidem, Codein, Ibuprofen was administered to patients.

\section{Statistical analysis}

Data presented in mean \pm standard deviation (SD). The normality of distribution was recognized using the Shapiro-Wilk test. Then, an independent t-test was used to evaluate the differences between performance variables in the baseline situation for pre-test data. The assumptions of a repeated measure ANOVA including the normality of data (Shapiro-Wilk's test), sphericity (Mauchly's test), and significant outliers was confirmed; then, values of all vaiabels, in except qulity of life (QOL), were analyzed. For QOL, the assumptions of one-way analysis of covariance (ANCOVA) including the normality of data (ShapiroWilk's test) and homogeneity of variance (box plot) was confirmed for variables that measured pre-and post-test; then, ANCOVA was used to analyze intergroup differences at the post-test values; and pre-test values were considered as a co-variant. Paired sample test was used to analyze differences within groups. The independent t-test was used to compare mean based on percentage. The effect size (ES) was also calculated as the change score divided by the SD of the change score to examine the magnitude of differences while controlling for the influence of the sample size, with 0.2 considered as a small ES, 0.5 as a moderate ES and $>0.8$ as a large ES. The significance level was set at $p \leq 0.05$ for all statistical analyses. The significance level was considered as $P \leq 0.05$. Data analysis was performed using SPSS software (SPSS, IBM, Chicago, IL, USA).

\section{Results}

Based on independent t-test analysis, there were no significant differences between both group in all variables in the pre-test $(p>0.05)$, in except QOL $(p=0.002, t=3.729)$ that ANCOVA was used to analysis QOL.

A repeated measure ANOVA with a Sphericity assumed correction determined that there was no a significant difference in weight, WHR, and BMI in control (CON) and experimental (EXP) groups with small effect sizes when the interaction of time $\times$ group for mean values was considered $(F[1,16]=1.448, p=$ $0.256, d=0.083),(F[1,16]=0.000, p=1.00, d=0.00)$, and $(F[1,16]=1.852, p=0.192, d=0.104)$, respectively). In addition, in both CON and EXP groups, the paired sample test analysis did not showed a 
significant difference in weight ( $t=-0.137, p=0.895$ and $t=0.68, p=0.517$ respectively), WHR $(t=0.607, p$ $=0.563$ and $t=1.861, p=0.096$ respectively), and $\mathrm{BMI}(\mathrm{t}=-0.244, \mathrm{p}=0.814$ and $\mathrm{t}=1.782, \mathrm{p}=0.104$ respectively) from pre- to post- test (see Fig. 1 and Table 3 ). Based on percentage changes, independent ttest outputs did not show a significant difference in weight $(p=0.206$ and $d=16)$, WHR $(p=0.0 .981$ and $d=16)$, and BMI $(p=0.206$ and $d=16)$ between CON and EXP groups.

The obtained results from special factors of migraine disease namely migraine indices including; intensity (INT), frequency (FRQ), and duration (DUR), a repeated measure ANOVA with a Sphericity assumed correction determined that there was a significant difference in INT, FRQ, and DUR in CON and EXP groups with large effect sizes when the interaction of time $\times$ group for mean values was considered $(F[1,16]=6.202, p=0.024, d=0.279),(F[1,16]=14.87, p=0.001, d=0.482)$, and $(F[1,16]=4.661, p=$ $0.046, d=0.226$ ), respectively). In addition, the paired sample test analysis showed a significant difference in migraine indices including INT $(t=3.214, p=0.011), F R Q(t=4.249, p=0.002)$, and DUR $(t=$ $2.848, p=0.019)$, from pre- to post- test, although there was no significant difference in migraine indices in CON group ( $p>0.05$ ) from pre- to post- test (see Fig. 2 and Table 3). Based on percentage changes, independent t-test outputs showed a significant difference in migraine indices including INT $(p=0.045, d$ $=16)$, WHR $(p<0.001, d=16)$, and BMI $(p=0.024$ and $d=16)$ between CON and EXP groups. 
Table 3

the parameters obtained from pre- and post-training protocol

\begin{tabular}{|c|c|c|c|c|c|}
\hline \multirow{2}{*}{$\begin{array}{l}\text { Groups } \\
\text { Variables }\end{array}$} & \multirow[t]{2}{*}{$\operatorname{CON}(\mathrm{N}=8)$} & \multirow[t]{2}{*}{$\operatorname{EXP}(\mathbb{N}=10)$} & \multicolumn{3}{|l|}{ p-value (ES) } \\
\hline & & & time & group & $\mathbf{T} \times \mathbf{g}$ \\
\hline \multicolumn{6}{|l|}{ weight } \\
\hline Pre-test & $60 \pm 9.30$ & $64.11 \pm 13.18$ & $0.33(0.057)$ & $0.52(0.026)$ & $0.24(0.083)$ \\
\hline Post-test & $60.07 \pm 9.34$ & $63.35 \pm 13.83$ & & & \\
\hline$\%$ changes & 0.12 & -1.18 & & & \\
\hline \multicolumn{6}{|l|}{ WHR } \\
\hline Pre-test & $0.76 \pm 0.03$ & $0.75 \pm 0.04$ & $0.22(0.091)$ & $0.36(0.052)$ & $1.00(000)$ \\
\hline Post-test & $0.76 \pm 0.04$ & $0.74 \pm 0.04$ & & & \\
\hline$\%$ changes & -0.64 & -0.66 & & & \\
\hline \multicolumn{6}{|l|}{ BMI } \\
\hline Pre-test & $23.04 \pm 4.15$ & $25.03 \pm 4.43$ & $0.33(0.058)$ & $0.39(0.045)$ & $0.19(0.104)$ \\
\hline Post-test & $23.09 \pm 4.26$ & $24.72 \pm 4.62$ & & & \\
\hline$\%$ changes & 0.21 & -1.24 & & & \\
\hline \multicolumn{6}{|c|}{ Headache intensity } \\
\hline Pre-test & $53.75 \pm 26.73$ & $67.70 \pm 37.59$ & $0.011(0.343)$ & $0.72(0.008)$ & $0.024 \rrbracket(0.279)$ \\
\hline Post-test & $50.87 \pm 15.44$ & $28.80 \pm 25^{\star}$ & & & \\
\hline$\%$ changes & -5.34 & -57.45 & & & \\
\hline \multicolumn{6}{|c|}{ Headache frequency } \\
\hline Pre-test & $10.25 \pm 2.91$ & $13.20 \pm 4.49$ & $0.017(0.309)$ & $0.60(0.017)$ & $0.001 \rrbracket(0.482)$ \\
\hline Post-test & $11.37 \pm 3.54$ & $7 \pm 2.62^{\star}$ & & & \\
\hline$\%$ changes & 10.97 & -46.96 & & & \\
\hline \multicolumn{6}{|c|}{ Headache duration } \\
\hline Pre-test & $69.37 \pm 33.78$ & $125.60 \pm 84.84$ & $0.018(0.304)$ & $0.35(0.055)$ & $0.046 \rrbracket(0.226)$ \\
\hline Post-test & $61.12 \pm 26.69$ & $44 \pm 49.67^{\star}$ & & & \\
\hline$\%$ changes & -11.89 & -64.96 & & & \\
\hline
\end{tabular}




\begin{tabular}{|c|c|c|c|c|c|}
\hline \multirow{2}{*}{$\begin{array}{l}\text { Groups } \\
\text { Variables }\end{array}$} & \multirow[t]{2}{*}{$\operatorname{CON}(\mathrm{N}=8)$} & \multirow[t]{2}{*}{$\operatorname{EXP}(N=10)$} & \multicolumn{3}{|l|}{ p-value (ES) } \\
\hline & & & time & group & $\mathbf{T} \times \mathbf{g}$ \\
\hline Pre-test & $52 \pm 7.91$ & $40.80 \pm 4.75$ & $0.001(0.622)$ & $0.42(0.033)$ & $0.001 \rrbracket(0.698)$ \\
\hline Post-test & $49.87 \pm 9.17$ & $65.90 \pm 10.56^{*}$ & & & \\
\hline$\%$ changes & -4.08 & 61.51 & & & \\
\hline \multicolumn{6}{|l|}{ Chest press } \\
\hline Pre-test & $18.64 \pm 3.90$ & $20.84 \pm 5.09$ & $0.002(0.465)$ & $0.002(0.465)$ & $0.000 \otimes(0.984)$ \\
\hline Post-test & $18.26 \pm 4.71$ & $30.19 \pm 5.58^{\star} \dagger$ & & & \\
\hline$\%$ changes & -2.03 & 44.82 & & & \\
\hline \multicolumn{6}{|l|}{ Leg press } \\
\hline Pre-test & $113.27 \pm 23.67$ & $125.67 \pm 30.06$ & $0.000(0.568)$ & $0.001(0.507)$ & $0.000 \otimes(0.603)$ \\
\hline Post-test & $111.44 \pm 13.08$ & $176.36 \pm 20.22^{*}+$ & & & \\
\hline$\%$ changes & -1.61 & 40.33 & & & \\
\hline
\end{tabular}

Data are expressed as mean \pm SD. ES, effect size. CON, control group; EXP, experimental group; QOL, quality of life.

*significant difference within group; †significant difference between groups;

$\mathbb{Q}$ significant difference in time $\times$ group

There was similarity observed a statistically significant interaction in quality of life (QOL). ANCOVA analysis showed there were a significant intergroup differences at QOL $(F[1,16]=13.903, p=0.002, d=$ 0.465), in CON and EXP groups with large effect sizes. In addition, the paired sample test analysis showed a significant difference in QOL from pre- to post- test in EXP group ( $t=-6.953, p<0.001)$, Not in CONT group ( $p>0.05$ ) from pre- to post- test (see Fig. 2 and Table 3). Based on percentage changes, independent t-test outputs showed a significant difference in QOL $(p<0.001, d=16)$ between CON and EXP groups. It is worth mentioning that the rate of change percent in EXP group was $61.51 \%$.

Finally, as it is pointed out in Fig. 3 and Table $3, \mathrm{t} \times \mathrm{g}$ interaction in both chest and leg presses was significant $(p=0.001)$. A repeated measure ANOVA with a Sphericity assumed correction determined that there was a significant difference in upper body strength (chest press) and lower body strength (leg press) in CON and EXP groups with large effect sizes when the interaction of time $\times$ group for mean values was considered $((F[1,16]=44.446, p<0.001, d=0.735)$ and $(F[1,16]=24.301, p<0.001, d=$ $0.603)$, respectively). In addition, the paired sample test analysis showed a significant difference in chest press $(t=-8.646, p<0.001)$ and leg press $(t=-7.333, p<0.001)$ from pre- to post-test, but no significant difference for chest press and leg press was observed in CON group $(p>0.05)$ from pre- to post-test (see 
Fig. 3 and Table 3). Based on percentage changes, independent t-test outputs showed a significant difference in chest press $(p<0.001, d=16)$ and leg press $(p=0.001, d=16)$, between CON and EXP groups.

\section{Discussion}

This study indicated that RT was well endured without any evidenced escalation in migraine indices. Furthermore, the results documented an enhancement, not only in headache status, but also in other studied variables such as QOL and upper- and lower-body strength. According to current findings, an eight-week RT program could lower headache intensity, frequencies and duration by $57 \%, 46 \%$ and $65 \%$, respectively. Furthermore, such improving was observed in other indices, namely QOL, upper and lower body strength that was by $61 \%, 44 \%$, and $40 \%$, respectively.

Though there are no studies about the effect of RT on migraine headache, the existing literature about endurance training has shown some improvement in migraine indices (i.e., headache intensity and frequencies) and QOL. Hence, Pairo et al (4) have shown that eight weeks of moderate-intensity aerobic training lowered headache intensity, duration and frequency, in turn by $62.32 \%, 78.42 \%$, and $67.07 \%$. It also enhanced both the QOL and Vo2max. They attributed this improvement possibly to the endogenous opioids system, specifically to beta-endorphins. Beta-endorphin is an endogenous opioid that is produced by the anterior pituitary and results in analgesia by binding to pre and post-synaptic opioid receptors (4, 22). It probably prevents the release of Substance $P(S P)$ in the peripheral nervous system, thereby, decreasing the transmission of pain pathways, although it would be designed more research about SPmediated role in migraine patients, although Eslami et al recently have shown no significant increase in $\mathrm{SP}$ in migraines after high- and moderate intensity aerobic training (12). In contrast, in the CNS, it functions as presynaptic in impeding the release of GABA (22). It results in excess production of dopamine, which is associated with pleasure $(22,23)$. In earlier reports regarding migraine and exercise, it has been found that there are low levels of serum beta-endorphin in migraineurs.( $(8,22,24)$ This opioid is even lower in patients with chronic migraine $(23,24)$. The previous documents also indicated that severe and chronic exercise increases beta-endorphin levels, possibly leading to fewer headache days $(4,8,22$, 24). Future studies recommend evaluating endorphin levels in migraineurs undergoing RT programs to find whether chronic deviances in the endorphin systems are correctable via this type of exercise.

Calcitonin Gene-Related Peptide (CGRP) is a 37-amino acid neuropeptide widely distributed in the CNS and PNS where it is involved in vasodilation and sensory transmission $(4,8)$. Research indicates that the levels of CGRP are increased in venous blood throughout an attack of migraine. Additionally, stimulation of trigeminal nerves may release CGRP, thus reinforcing already existing vasodilatation and relaying nociceptive impulses to the CNS $(4,8)$. In 2012, Parnow et al studied the content of CGRP in trigeminal nerves in Wistar rats implemented in endurance, resistance, and strength training. Their results illustrated an increase in CGRP content due to strength training with maximum intensity. However, they did report any variation in CGRP content comparing endurance training and RT. The findings considered that exercise intensity probably plays a key element in modifying CGRP content. From this perspective, the 
low-level release of CGRP in the trigeminal nerve is probably because of moderate and low-intensity physical activity (14). In future, we will show CGRP levels in migraineurs undergoing aerobic exercise programs to discover the possible relationship between CGRP and migraine disorders in the patients.

Physical fitness has a positive effect on health and wellbeing (25). There is also an inverse relationship between migraine experience and physical fitness (25). Earlier studies have reported that there is possibly reverse correlation between migraine indices (i.e., pain severity, frequency, and duration) and physical fitness $(4,25,26)$. The present study showed that an eight-week period of RT/ three times a week led to an increase in strength (44\% increase in upper body strength and $40 \%$ in lower body strength). Since the lack of literature in this area, the mechanism of RT-related changes in muscle increase and migraine indices reduction are unknown, although our assumption was that blood circulation probably plays a main role in the body due to physical activity, in the form of RT. This situation would may mediate the unknown mechanism to reduce migraine indices and improve QOL and physiological elements. Further investigations, therefore, are warranted to examine the relationship between strength and migraine.

The results also illustrated that QOL increased by $61 \%$ following eight-week RT. This finding is in line with previous works that exercise is beneficial in upgrading QOL $(4,22,25,27)$. Exercise can manage several chronic pain conditions $(22,28)$. These conditions are depression, anxiety, sleep disturbances, and lack of confidence comorbid with migraine $(4,22,29)$. Several epidemiological studies have shown that migraine is often associated with several psychiatric disorders such as generalized anxiety disorder, major depressive disorder, anxiety disorder, bipolar disorder, and personality disorders $(4,22,30)$. Research further depicts that exercise could escalate self-esteem, also associated with migraine symptoms improvement $(22,29)$. Based on the evidence, it seems that both the psychological and physiological effects of the exercises are crucial in QOL improvement.

\section{Conclusions}

This investigation indicates that patients suffering from migraine headaches well endured an exercise intervention. our results, therefore, demonstrate that an eight-week RT program improves QOL and upperand lower-body strength and reduces migraine indices (duration, intensity, and frequency) in women with migraine disorder. Hence, a RT program may be considered as an alternative therapy or augmentative complement to establish interventions for migraine. Based on this RT-related changes, it would may open new insight for researchers to be interesting in new research area in migraine patients with new approach in considering positive effect of resistance training. In this study, there was some limitations. For example, all subjects were female, and this was a limitation. Our suggestion for future research is to conduct studies on men, although the main limitation was about insufficient research that investigated effect of resistance training on people with migraine disordes. Future study would be designed to explain involved different mechanism in current society.

\section{Abbreviations}


1RM: One-Repetition Maximum

BMl: Body Mass Index

CGRP: Calcitonin Gene-Related Peptide

CSD: Cortical Spreading Depression

Duration: DUR

Frequency: FRQ

HIT: Headache Impact Tests

ICHD: International Classification of Headache Disorders

IHS: International Headache Society

Intensity: INT

QOL: Quality of Life

RT: Resistance Training

SED: Sedentary

VAS: Visual Analog Scale

WHR: Waist to Hip Ratio

\section{Declarations}

Acknowledgment: We sincerely thank the subjects for their dedication and participation in this study. We also gratefully acknowledge the technical assistance of Ph.D. students to monitor in practical training, Mrs. Zahra Pairo, Ms. Afsaneh Astinchap, and Ms. Jaleh Amirian.

Funding: This work was supported by the Medical Sciences University, Kermanshah, Iran (No.1394.15)

Conflict of interest: All authors declare no conflicts of interest.

Availability of data and material: All data are available and transparency.

Code availability: All data were analyzed using SPSS 22.0 statistical software and presented as means \pm SD.

Authors' contributions: AP contributed in designing research and final edition, MA contributed in literature searching, train subjects, and data collection. OR contributed in preparation manuscript, BK contributed in 
discussion preparation, and, PS contributed as supervisor and Neurologist to Migraine patients.

Ethics approval: The Medical Ethics Committee formally approved this research, Medical Sciences University, Kermanshah, Iran (ethical code: IR.kums.rec.1394.15), and it was registered at the Iranian Registry of Clinical Trials (IRCT2015100910824N2).

Consent to participate: All patients were requested to fill out the consent form.

Consent for publication: All authors are consent to publish the manuscript.

\section{References}

1. Durstine JL, Painter P, Franklin BA, Morgan D, Pitetti KH, Roberts SO (2000) Physical activity for the chronically III and disabled. Sports Med 30(3):207-219

2. Varkey E, Grüner Sveälv B, Edin F, Ravn-Fischer A, Cider $\AA$ (2017) Provocation of migraine after maximal exercise: A test-retest study. Eur Neurol 78(1-2):22-27

3. Varkey E, Cider A, Carlsson J, Linde M (2009) A Study to evaluate the feasibility of an aerobic exercise program in patients with migraine. Headache 49(4):563-570

4. Pairo Z, Parnow A, Sari-aslani P, Eslami R (2016) Effect of Moderate Intensity Aerobic Exercise on Migraine Headache Indexes and Quality of Life Improvement in Women with Migraine. Quarterly of Horizon of Medical Sciences 22:291-297

5. Busch V, Gaul C (2008) Exercise in migraine therapy - Is there any evidence for efficacy? A critical review. Headache 48(6):890-899

6. Eftekhari S, Edvinsson L (2010) Possible sites of action of the new calcitonin gene-related peptide receptor antagonists. Therapeutic Advances in Neurological Disorders 3(6):369-378

7. Varkey E (2012) On the prevention of migraine-focus on exercise and the patient's perspective. University of Gothenburg, Gothenburg

8. Hindiyeh NA, Krusz JC, Cowan RP. Does exercise make migraines worse and tension type headaches better? Current Pain and Headache Reports. 2013;17(12)

9. Schwartz JS, Song P, Blitzer A. Headache. Therapeutic Uses of Botulinum Toxin. 2007:91-108

10. Gil-Martínez A, Kindelan-Calvo P, Agudo-Carmona D, Muñoz-Plata R, López-de-Uralde-Villanueva I, La Touche R (2013) Therapeutic exercise as treatment for migraine and tension-type headaches: A systematic review of randomised clinical trials. Revista de Neurologia 57(10):433-443

11. Totzeck A, Unverzagt S, Bak M, Augst P, Diener HC, Gaul C. Aerobic endurance training versus relaxation training in patients with migraine (ARMIG): study protocol for a randomized controlled trial. Trials. 2012;13

12. Eslami R, Parnow A, Pairo Z, Nikolaidis P, Knechtle B (2021) The effects of two different intensities of aerobic training protocols on pain and serum neuro-biomarkers in women migraineurs: a randomized controlled trail. Eur J Appl Physiol 121(2):609-620 
13. Barber M, Pace A (2020) Exercise and migraine prevention: A review of the literature. Curr Pain Headache Rep 24(8):1-7

14. Parnow AH, Gharakhanlou R, Eslami R (2012) Effects of physical activity on calcitonin gene-related peptide content at trigeminal ganglion nerve in wistar rats. Journal of Mazandaran University of Medical Sciences 22(90):25-31

15. La Touche R, Fernández Pérez JJ, Proy Acosta A, González Campodónico L, Martínez García S, Adraos Juárez D et al (2020) Is aerobic exercise helpful in patients with migraine? A systematic review and meta-analysis. Scand J Med Sci Sport 30(6):965-982

16. Song T-J, Chu MK (2021) Exercise in treatment of migraine including chronic migraine. Curr Pain Headache Rep 25(3):1-11

17. Faul F, Erdfelder E, Lang A-G, Buchner A (2007) G* Power 3: A flexible statistical power analysis program for the social, behavioral, and biomedical sciences. Behavior research methods 39(2):175191

18. Marchese R, Taylor J, Fagan K (2019) The essential guide to fitness: for the fitness instructor. Cengage Learning Australia

19. Brzycki M (1989) A practical approach to strength training. Masters Press Grand Rapids, MI

20. Yang M, Rendas-Baum R, Varon SF, Kosinski M (2011) Validation of the Headache Impact Test (HIT$6^{\mathrm{TM}}$ ) across episodic and chronic migraine. Cephalalgia 31(3):357-367

21. De Wilde JA, Van Dommelen P, Middelkoop BJC. Appropriate body mass index cut-offs to determine thinness, overweight and obesity in South Asian children in The Netherlands. PLoS ONE. 2013;8(12)

22. Amin FM, Aristeidou S, Baraldi C, Czapinska-Ciepiela EK, Ariadni DD, Di Lenola D et al (2018) The association between migraine and physical exercise. J Headache Pain 19(1):83

23. Misra UK, Kalita J, Tripathi GM, Bhoi SK (2013) Is $\beta$ endorphin related to migraine headache and its relief? Cephalalgia 33(5):316-322

24. Köseoglu E, Akboyraz A, Soyuer A, Ersoy A (2003) Aerobic exercise and plasma beta endorphin levels in patients with migrainous headache without aura. Cephalalgia 23(10):972-976

25. Krøll LS, Hammarlund CS, Linde M, Gard G, Jensen RH (2018) The effects of aerobic exercise for persons with migraine and co-existing tension-type headache and neck pain. A randomized, controlled, clinical trial. Cephalalgia 38(12):1805-1816

26. Darabaneanu S, Overath $\mathrm{CH}$, Rubin D, Lüthje S, Sye W, Niederberger U et al (2011) Aerobic exercise as a therapy option for migraine: A pilot study. Int J Sports Med 32(6):455-460

27. Jackson W, Zale EL, Berman SJ, Malacarne A, Lapidow A, Schatman ME et al (2019) Physical functioning and mindfulness skills training in chronic pain: A systematic review. Journal of Pain Research 12:179-189

28. Daenen L, Varkey E, Kellmann M, Nijs J (2015) Exercise, not to exercise, or how to exercise in patients with chronic pain? Applying science to practice. Clin J Pain 31(2):108-114 
29. Irby MB, Bond DS, Lipton RB, Nicklas B, Houle TT, Penzien DB (2016) Aerobic Exercise for Reducing Migraine Burden: Mechanisms, Markers, and Models of Change Processes. Headache 56(2):357369

30. Bigal M, Krymchantowski AV, Lipton RB (2009) Barriers to satisfactory migraine outcomes. what have we learned, where do we stand?: Views and perspectives. Headache 49(7):1028-1041

\section{Figures}
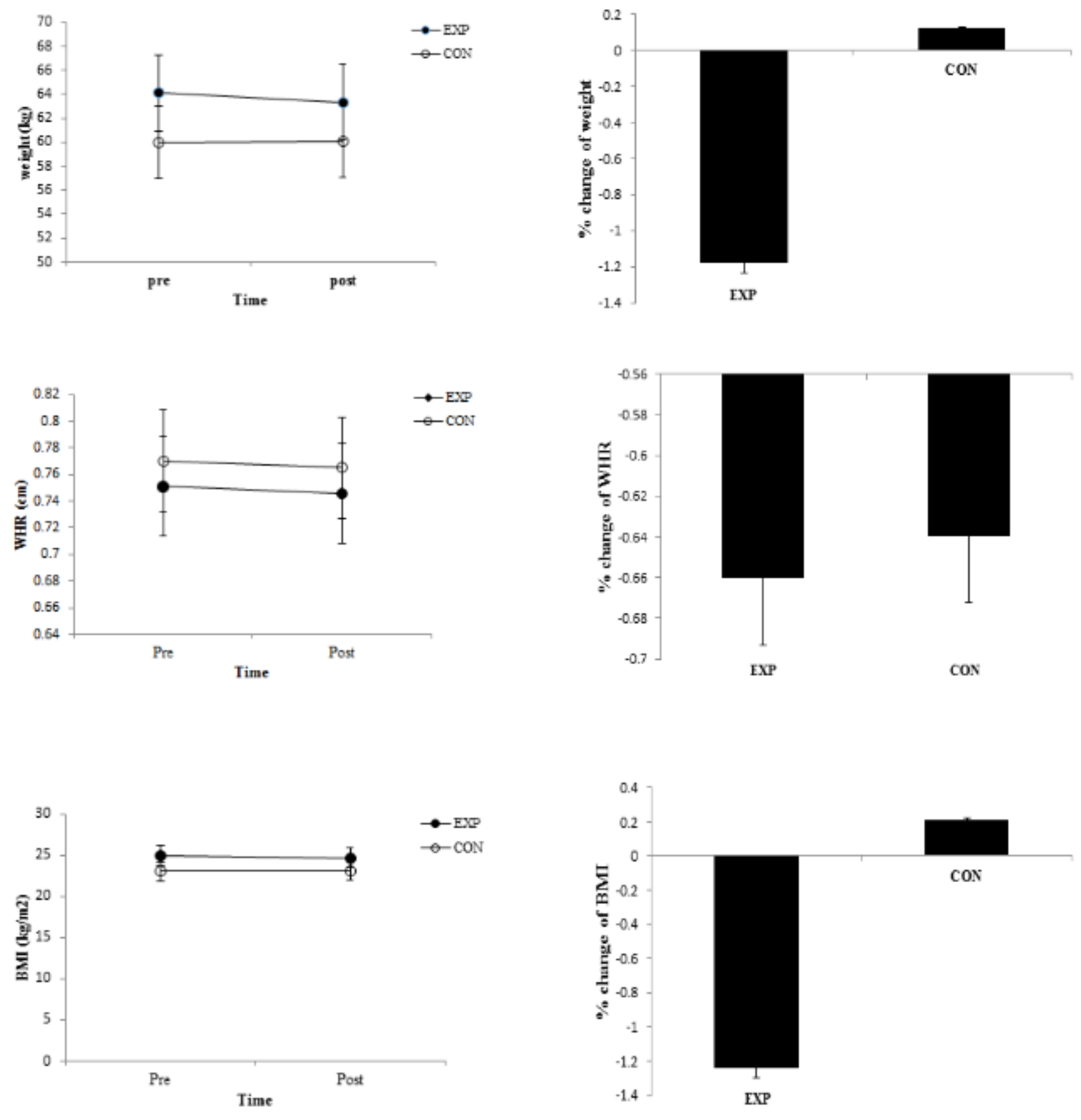

Figure 1 
Diagram shows the measurements before and after 8-week resistance training protocol in control and experimental groups. The change percent of indices is depicted in right-hand position in both groups. CON, control group; EXP, experimental group; WHR, waist-hip ratio; BMI, body mass index.
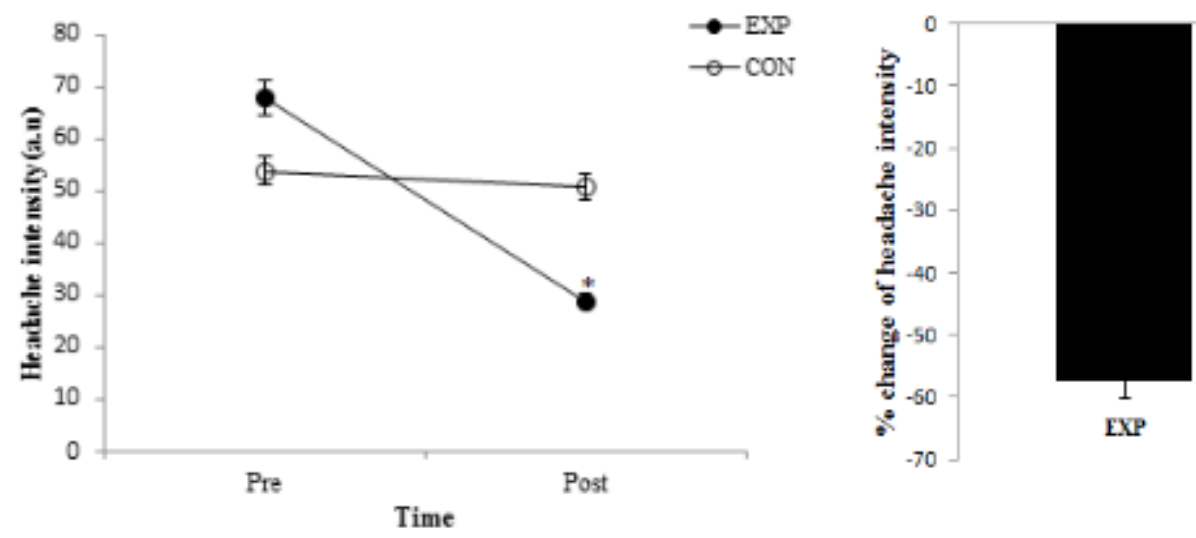

cos
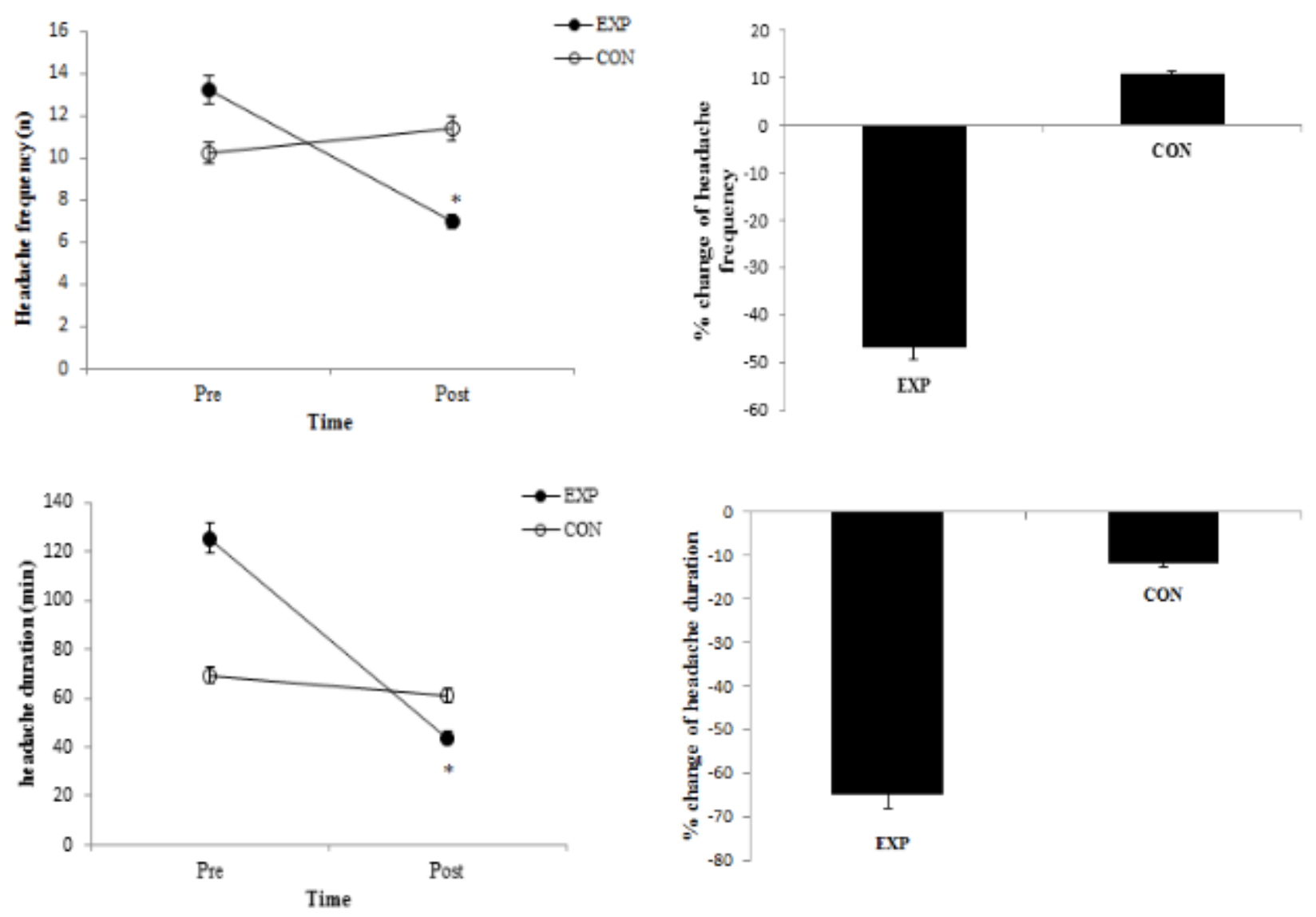

$\cos$

Figure 2

Diagram shows the measurements before and after 8-week resistance training protocol in control and experimental groups. The change percent of indices is depicted in right-hand position in both groups. CON, control group; EXP, experimental group. *significant difference within group. 

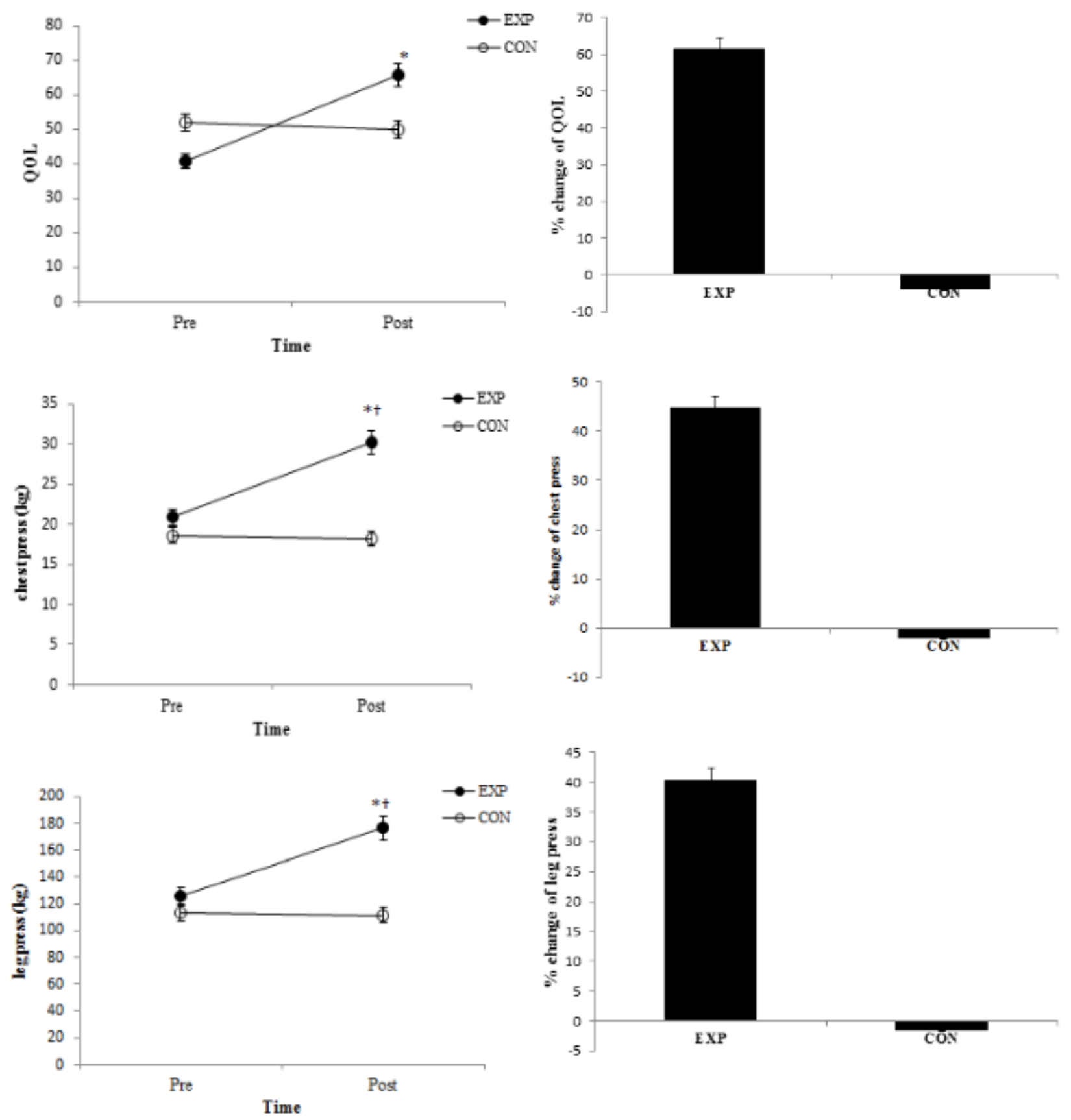

Figure 3

Diagram shows the measurements before and after 8-week resistance training protocol in control and experimental groups. The change percent of indices is depicted in right-hand position in both groups. CON, control group; EXP, experimental group; QOL, quality of life. *significant difference within group; tsignificant difference between groups. 